\title{
Stammzellbiologie
}

\section{Sexualdimorphismen adulter Stammzellen}

MADLEN MERTEN, JOHANNES F. W. GREINER, CHRISTIAN KALTSCHMIDT, BARBARA KALTSCHMIDT

LEHRSTUHL FÜR ZELLBIOLOGIE, UNIVERSITÄT BIELEFELD

\section{Neurodegenerative diseases like Parkinson's disease differ between} the sexes in severity and occurrence. Next to hormons, increasing evidence suggests stem cell-intrinsic mechanisms to account for pathologic sex-specific differences. Here, we discuss such sex-related intrinsic mechanisms and sex-specific differences in adult stem cells, neuronal development and neuroprotection. The reviewed observations emphazise the importance of considering sexual dimorphisms for medical treatment strategies.

DOI: $10.1007 / \mathrm{s} 12268-020-1333-2$

(C) Die Autoren 2020
Für unsere Forschung verwenden wir ITSCs (inferior turbinate stem cells), welche zu den Neuralleisten-abgeleiteten Stammzel- len (neural crest-derived stem cells, NCSCs) gehören und aus der unteren Nasenmuschel (inferior turbinate) isoliert werden (Abb. 1).

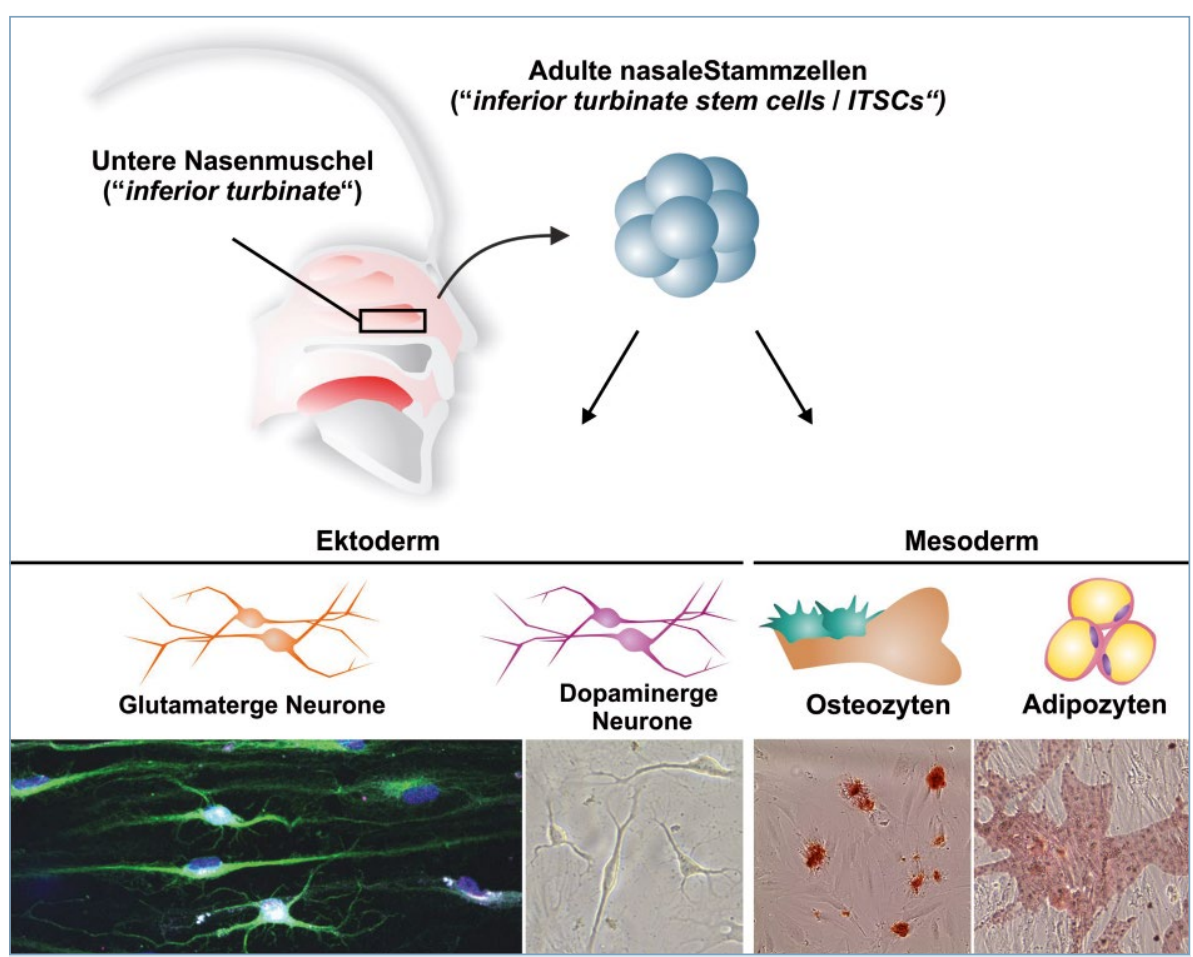

A Abb. 1: ITSCs (inferior turbinate stem cells) werden aus dem Gewebe der unteren Nasenmuschel isoliert und sind in der Lage, sich in ektodermale Zelltypen, wie glutamaterge und dopaminerge Neuronen, und mesodermale Zelltypen, wie Osteo- und Adipocyten, zu differenzieren (teilweise modifiziert nach [9]).
Das zur Isolation der Stammzellen verwendete Gewebe wird in einer routinemäßigen Operation bei obstruktivem Schnarchen entnommen [1]. NCSCs verdanken ihren Namen dem entwicklungsbiologischen Ursprung dieser Stammzellen aus der Neuralleiste. Diese entsteht während der Embryonalentwicklung zwischen dem Ektoderm und dem Neuralrohr, aus welchem sich das Zentralnervensystem entwickelt. Von der Neuralleiste aus migrieren die Stammzellen in den sich entwickelnden Embryo und tragen nach erfolgter Migration wesentlich zur weiteren embryonalen Entwicklung bei, indem sie in verschiedenste Zelltypen des Ekto- und Mesoderms differenzieren (Abb. 1). Mithilfe von bestimmten Supplementen, wie Nährund Signalstoffen, können Stammzellen zur Differenzierung angeregt werden. Hierbei wurde festgestellt, dass ITSCs bereits in diesem frühen Stadium geschlechtsspezifische Unterschiede auf zellulärer Ebene zeigen. Aus männlichen Patienten isolierte ITSCs differenzieren im Vergleich zu weiblichen ITSCs besser in Knochenvorläuferzellen (osteogene Differenzierung). Im Gegensatz hierzu differenzieren die ITSCs aus weiblichen Patienten schneller in Nervenzellen (neuronale Differenzierung) als männliche Zellen.

Die Rolle des Geschlechts bei der Differenzierung von Stammzellen zu Nervenzellen

Im Zuge der neuronalen Differenzierung können ITSCs erfolgreich in glutamaterge Neurone differenziert werden (Abb. 1). Werden die Zellen zusätzlich mit dem Hormon Erythropoetin kultiviert, sind bei den weiblichen Zellen nach zehn Wochen deutlich verlängerte Axone sowie stärkere Verzweigungen $\mathrm{zu}$ beobachten, was auf eine schnellere Reifung der Zellen hindeutet. Männliche Nervenzellen hingegen zeigen diese Veränderungen nicht (Abb. 2). Neben der Funktion als Wachstumsfaktor bei der Bildung von Erythrocyten werden dem Hormon auch neuroprotektive Eigenschaften zugesprochen. 
Während der Differenzierung von ITSCs zu dopaminergen Neuronen zeigt sich ebenfalls, dass weibliche ITSCs schneller reifen. Sie weisen im Vergleich zu den männlichen Neuronen neben einem stärkeren Neuritenwachstum auch eine höhere Verzweigung und eine höhere Expression der Thyrosinhydroxylase auf, einem Marker für reife dopaminerge Neurone. Behandelt man dopaminerge Neurone mit dem Neurotoxin 6-OH-Dopamin, zeigen männliche Neurone im Vergleich zu weiblichen eine höhere Sterblichkeit (Abb. 3, [2, 3]). Die dopaminergen Neurone spielen eine essenzielle Rolle bei Parkinson, da der Verlust dieser Neuronen die Ursache der Erkrankung darstellt. In Übereinstimmung mit unseren Daten lässt sich beobachten, dass Männer häufiger an Parkinson erkranken als Frauen [3]. Neben dem Neurotoxin wurde auch der Einfluss von oxidativem Stress in Neuronen untersucht. Dieser wird durch Zugabe von Wasserstoffperoxid induziert. Im Gegensatz zur Applikation des Neurotoxins führt der oxidative Stress zu einer höheren Sterblichkeit von weiblichen Neuronen im Vergleich zu ihren männlichen Gegenstücken (Abb. 3, [4]). Die Ursache dieses starken sexuellen Dimorphismus sind Gegenstand aktueller Studien und zum Teil auf den Transkriptions-

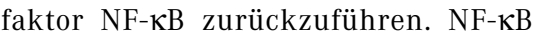
(nuclear factor , kappa-light-chain-enhancer' of activated B-cells) spielt eine zentrale Rolle in einer Vielzahl zellulärer Prozesse, wie Zellwachstum, Apoptose, Inflammation, Lernen und Gedächtnis oder Immunität. Die Aktivierung von NF- $\kappa B$ mithilfe des proinflammatorischen Cytokins Tumornekrosefaktor- $\alpha$ (TNF- $\alpha$ ) resultiert in einer signifikanten Neuroprotektion gegen oxidativen Stress in beiden Geschlechtern. In weiblichen Neuronen, die sensitiver gegen oxidativen Stress sind (Abb. 3), werden nach Aktivierung von NF-кB die Zielgene SOD2 und IGF2 zur Neuroprotektion hochreguliert, wogegen die Neuroprotektion in männlichen Neuronen durch Hochregulation des Gens PKA cat alpha gefördert wird [4]. Neben oxidativem Stress wurden auch geschlechtsspezifische Unterschiede bei Alkoholmissbrauch festgestellt. Hierbei können Hirnschäden im Zusammenhang mit Alkoholmissbrauch bei Frauen verstärkt und schneller auftreten, wobei Männer eine relative Neuroprotektion aufweisen. Weiterhin sind Frauen anfälliger für alkoholinduzierte Schädigungen wie Zirrhosen oder Kardiomyopathien und zeigen einen erschwerten Alkoholentzug im Gegensatz zu Männern [3]. Auch in einem Mausmodell wurde gezeigt, dass weibliche Mäuse sensitiver auf Ethanol reagieren (Abb. 3). Nach Alkoholexposition und Alkoholentzug werden jeweils bestimmte Gene in weiblichen Mäusen höher exprimiert als bei

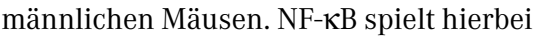
in beiden Geschlechtern eine essenzielle Rolle, jedoch wurden zwischen den Geschlechtern keine der NF-кBabhängigen Gene gemeinsam reguliert. Weibliche Mäuse zeigen unter anderem eine Hochregulation der Cyto- und Chemokine TNF- $\alpha$, Interleukin-6 (IL-6), Eotaxin und CC-Chemokin-Ligand 5 (CCL5), die proinflammatorische Reaktionen regulieren [5]. Diese Erkenntnisse bestätigen die bereits beobachtete proinflammatorische Toxizität und Degeneration in weiblichen Zellen. Im Gegensatz dazu zeigen männliche Mäuse eine Herunterregulierung der Chemokine CCL28, CCL5 und des Rezeptors CXCR5, was zu Immunsuppression und einer relativen Neuroprotektion führt $[3,5]$. Bezüglich NF-אB wurden bei Mäusen zudem geschlechtsspezifische Unterschiede im Transkriptom von Mikroglia entdeckt. 79 Prozent von 95 inflammatorischen NF-кB-Zielgenen zeigen eine erhöhte Expression in männlichen Mikroglia, was männliche Mäuse anfälliger für entzündliche Reaktionen macht (Abb. 3). Im Vergleich hierzu zeigten weibliche Mikroglia neuroprotektive Eigenschaften, die auch in männlichen Mäusen beobachtet werden, nachdem man ihnen weibliche Mikroglia injiziert hat [6].

Sexualdimorphismen lassen sich ebenfalls im Rahmen der Neurogenese beobachten. Bei diesem Prozess werden aus Stammzellen neue Nervenzellen gebildet. Neurogenese findet nicht nur während der Embryonalentwicklung, sondern auch im adulten Organismus statt. Die Stammzellnischen der adulten Neurogenese sind die subventrikuläre Zone und der Gyrus dentatus, welcher einen Teil des Hippocampus darstellt. Die geschlechtsabhängige Regulation der Neurogenese im Hippocampus wird durch zirkulierende Sexualhormone vermittelt [7]. Dabei zeigen Frauen eine erhöhte Neurogenese im 

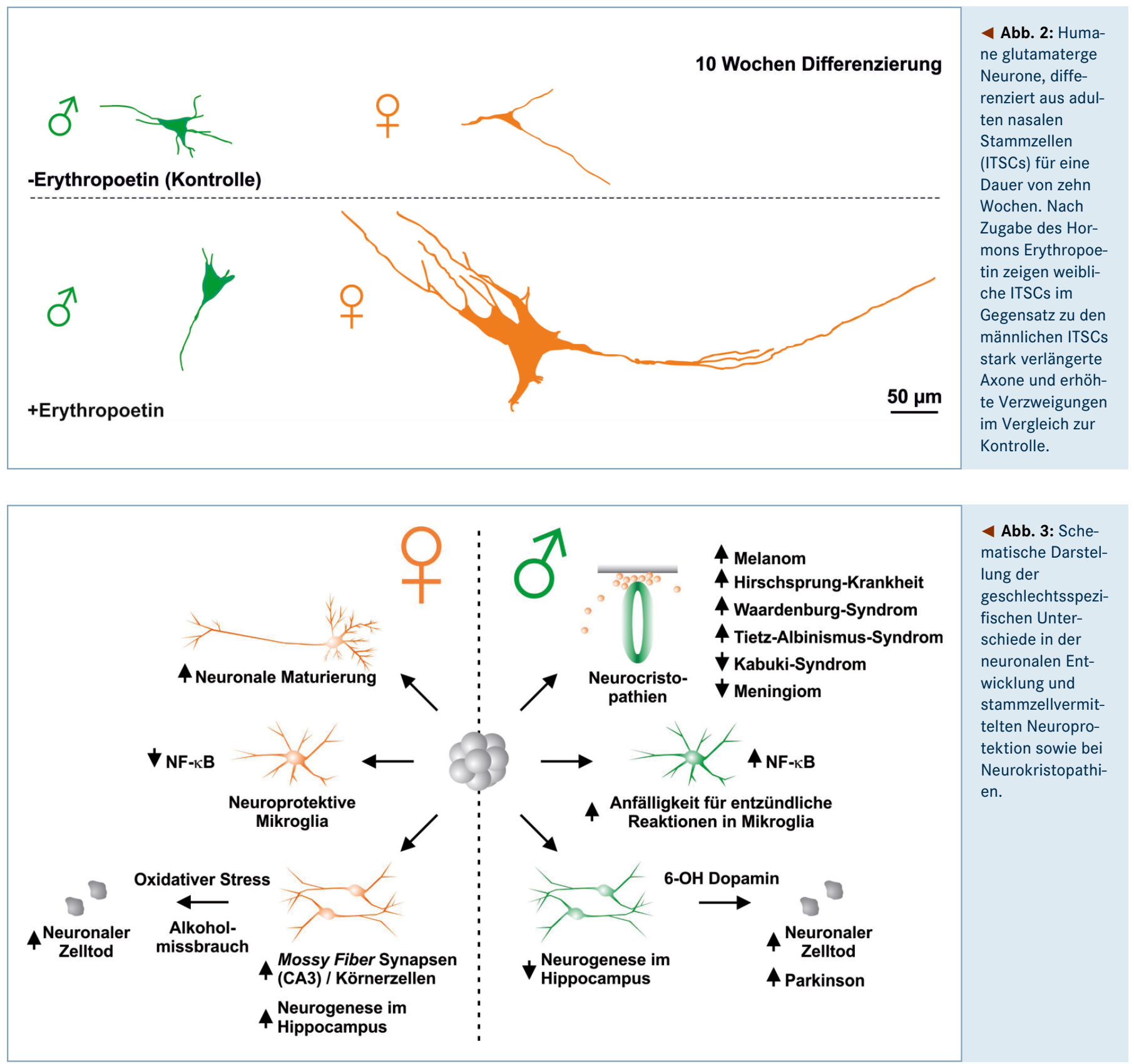

Hippocampus, nämlich eine höhere Anzahl Moosfaser-Synapsen in der CA3-Region und mehr Körnerzellen, wohingegen jedoch die Degenerationsrate höher ist (Abb. 3, [8]).

Neben dem Zentralnervensystem zeigen Untersuchungen auch geschlechtsspezifische Unterschiede bei der Entwicklung des peripheren Nervensystems, das größtenteils aus migrierenden Neuralleistenzellen entsteht. Bei der embryonalen Entwicklung von Mäusen besiedeln Neuralleistenzellen das Innere des Eierstocks und differenzieren dort in Neurone und Gliazellen. Diese bilden das ganze neurale Netzwerk der Eierstöcke. Im Vergleich dazu wird das Hodengewebe nicht durch Neuralleistenzellen besiedelt [3].
Geschlechtsspezifische Unterschiede bei Entwicklungsstörungen der Neuralleiste

Bei der humanen Entwicklung der Neuralleiste kann es zu Störungen kommen, den Neurokristopathien. Zu den geschlechtsspezifischen Neurokristopathien zählen der Morbus Hirschsprung, das WaardenburgSyndrom, Meningiome und Melanome, das Tietz-Albinismus-Taubheits-Syndrom und das Kabuki-Syndrom (Abb. 3). Der Morbus Hirschsprung, welcher häufiger bei Männern auftritt, ist durch einen fehlerhaften Aufbau des Nervensystems im Darm charakterisiert. Ursache hierfür ist die fehlende Migration von Ganglienvorläuferzellen aus der Neuralleiste. Durch die fehlenden Ganglienzellen im Darm ist die Darmperistaltik gestört, was zur Obstruktion (Verengung) führt. Viele der Neuropathien, wie das Waardenburg-Syndrom, sind mit dem Morbus Hirschsprung assoziiert. Auch das Waardenburg-Syndrom weist eine geschlechtsspezifische Prävalenz bei Männern auf. Neuralleisten-abgeleitete Tumoren, wie das Meningiom, treten häufiger bei Frauen auf, während Melanome häufiger bei Männern zu finden sind. Neben diesen Tumoren zeigen sich geschlechtsspezifische Unterschiede in der Prävalenz des Kabuki-Syndroms, das häufiger bei Frauen auftritt und sich durch kraniofaziale Defekte, Herzfehler und post- 
natale Wachstumsverzögerungen auszeichnen [3].

Die geschlechtsspezifischen Unterschiede in Neurokristopathien und neurodegenerativen Erkrankungen wie Parkinson sollten vor allem im therapeutischen Kontext stärker berücksichtigt und genauer untersucht werden. Vor allem die Unterschiede auf Stammzellebene können sich als entscheidender Faktor für zukünftige Behandlungsstrategien herausstellen.

\section{Literatur}

[1] Hauser S, Widera D, Qunneis F et al. (2012) Isolation of novel multipotent neural crest-derived stem cells from adult human inferior turbinate. Stem Cells Dev 21:742756

[2] Lieb K, Andrae J, Reisert I et al. (1995) Neurotoxicity of dopamine and protective effects of the NMDA receptor antagonist AP-5 differ between male and female dopaminergic neurons. Exp Neurol 134:222-229

[3] Greiner JFW, Merten M, Kaltschmidt C et al. (2019) Sexual dimorphisms in adult human neural, mesoderm-derived, and neural crest-derived stem cells. FEBS Lett 593:3338-3352

[4] Ruiz-Perera LM, Schneider L, Windmoller BA et al. (2018) NF-kappaB p65 directs sex-specific neuroprotection in human neurons. Sci Rep 8:16012

[5] Wilhelm CJ, Hashimoto JG, Roberts ML et al. (2014) Understanding the addiction cycle: a complex biology with distinct contributions of genotype vs. sex at each stage. Neuroscience 279:168-186
[6] Villa A, Gelosa P, Castiglioni L et al. (2018) Sexspecific features of microglia from adult mice. Cell Rep 23:3501-3511

[7] Hillerer KM, Neumann ID, Couillard-Despres S et al. (2013) Sex-dependent regulation of hippocampal neurogenesis under basal and chronic stress conditions in rats. Hippocampus 23:476-487

[8] Madeira MD, Paula-Barbosa MM (1993)

Reorganization of mossy fiber synapses in male and female hypothyroid rats: a stereological study. J Comp Neurol 337:334-352

[9] Greiner JF, Gottschalk M, Fokin M et al. (2019) Natural and synthetic nanopores directing osteogenic differentiation of human stem cells. Nanomedicine 17:319328

Funding: Open Access funding provided by Projekt DEAL.

Open Access: Dieser Artikel wird unter der Creative Commons Namensnennung 4.0 International Lizenz veröffentlicht, welche die Nutzung, Vervielfältigung, Bearbeitung, Verbreitung und Wiedergabe in jeglichem Medium und Format erlaubt, sofern Sie den/die ursprünglichen Autor(en) und die Quelle ordnungsgemäß nennen, einen Link zur Creative Commons Lizenz beifugen und angeben, ob Änderungen vorgenom Drittm. Dia desem An une Lizenz, sofern sich aus der Abbildungslegende nichts anderes ergibt. Sofern das betreffende Material nicht unter der genannten Creative Commons Lizenz steht und die betreffende Handlung nicht nach gesetzlichen Vorschriften erlaubt ist, ist für die oben aufgeführten Weiterverwendungen des Materials die Einwilligung des jeweiligen Rechteinhabers einzuholen. Weitere Details zur Lizenz entnehmen Sie bitte der Lizenzinformation auf http://creativecommons.org/licenses/by/4.0/

Korrespondenzadresse:

Prof. Dr. Barbara Kaltschmidt

Universität Bielefeld

Fakultät für Biologie

Zellbiologie der Tiere

Universitätsstraße 25

D-33615 Bielefeld

barbara.kaltschmidt@uni-bielefeld.de

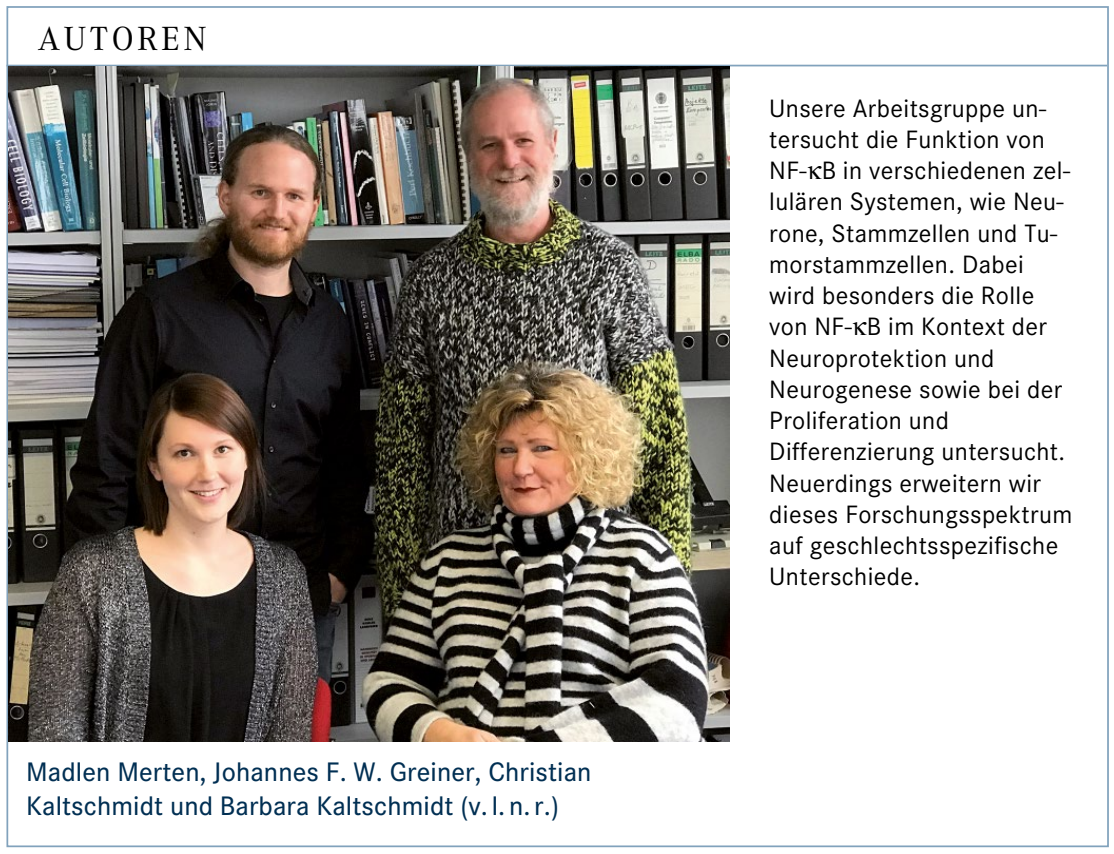

